\title{
Using Sequential Debugging Techniques with Massively Parallel Programs
}

\author{
Christian Schaubschläger, Dieter Kranzlmüller, and Jens Volkert \\ GUP, Joh. Kepler University Linz \\ Altenbergerstr. 69 \\ A-4040 Linz, Austria, Europe \\ schaubschlaeger@gup.uni-linz.ac.at
}

\begin{abstract}
Debugging is a crucial part of the software development process. Especially massively-parallel programs impose huge difficulties to program analyis and debugging due to their higher complexity compared to sequential programs. For debugging and analysing parallel programs there are several tools available, but many of these fail in case of massively-parallel programs with potentially thousands of processes.

In this work we introduce the single process debugging strategy, a scalable debugging strategy for massively-parallel programs. The goal of this strategy is to make debugging large scale programs as simple and straight-forward as debugging sequential programs. This is achieved by adapting and combining several techniques which are well known from sequential debugging. In combination, these techniques give the user the possibility to execute and investigate small fractions of a possibly huge parallel program, without having to (re-)execute the entire program.
\end{abstract}

\section{Introduction}

In the past years we can see a drastic increase of hardware and software complexity, especially in the field of high performance computing. In order to satisfy the steadily increasing needs of the supercomputing community, computer manufacturers try to increase the degree of parallelism of the hardware. This can be observed on several system layers, starting at processor level. With the integration of more than one processor core on one CPU very compact SMP systems with a relatively high number of processor cores can be built.

Since scalability of SMP systems is limited to several dozens of processor cores, NUMA systems or clustering techniques are used to increase the number of processor cores further. NUMA systems scale well up to a few thousand of processor cores. Using cluster computing techniques, SMPs or NUMAs are connected to larger systems with possibly more than a hundredthousand processor cores.

In recent years we see also software-sided approaches to build systems which exceed the borders of large clusters. Such ideas evolved originally from distributed computing, where distributed resources were used to solve a given problem, like in the SETI@ home Project or in the GIMPS Project, just to name two popular. Researchers work on advanced middleware to build so called GRID Systems [1]. Such systems improve and 
extend the idea of distributed computing in terms of resource allocation, scheduling, security, and many others. One goal is to build a possibly global metacomputer with hundreds of thousands of processors.

With the increase of the available computing power, one can also observe an increase of software complexity. To utilise the hardware resources on such large scale computers as good as possible, usually massively-parallel programs are used. Due to their size (in terms of number of processes, memory consumption, disk space, etc.) such programs impose big problems especially when it comes to program analysis and debugging. Most of the available tools in that area cannot handle such huge programs well enough. A common approach is to debug not the full scale program, but try to work on a smaller problem size, use less processors, etc. However, this approach does not work always, since some errors occur only in the full scale case. Therfore there is a strong need for new strategies in program analysis and debugging of massively-parallel programs. In this paper we propose a novel strategy, which tries to hide the high complexity of the program under analysis from the user, thus making it easier to handle.

The rest of the paper is organised as follows. In section 2 we describe important aspects of program analysis in general and problems of analysing massively-parallel program in particular. In section 2.3 we give a brief overview about related work in this area. In section 3 the single process debugging strategy is introduced and described. Section 4 concludes the paper.

\section{Program Analysis}

\subsection{Debugging Techniques}

Program analysis is an important part of the software life-cycle, because an effective analysis and proper use of analysis results can reduce the costs of software development and maintainance enormously. Three main aspects of program analysis are performance analysis, testing, and error debugging. In our work the focus lies on debugging, and debugging strategies respectively.

Many well known techniques and methods for program debugging have been developed originally for debugging sequential programs. Amongst others we mention the following, which are important for our debugging strategy: Single-step debugging, Cyclic debugging, Checkpointing, Breakpointing, and Control flow analysis and Program slicing. The reasons why these techniques cannot be applied to parallel or massively parallel programs, respectively, ad-hoc, and why such programs impose enormous difficulties on the debugging process, is described in the following sections.

\subsection{Massively Parallel Programs}

The difficulties in debugging massively parallel programs stem from their sheer size, both in execution time, as well as in space (number of processes, memory consumption, etc). A common practice to get information about a program's execution is to instrument the program, i.e. to insert additional monitoring code, which collects information during execution. This data is subject to analysis, either post-mortem or online.

This approach works well for small and medium-size programs, but it becomes impractical for large programs, since the amount of collected data is too big. There are hardly 
any analysis techniques to extract useful information, and even more important, there are almost no proper techniques to visualize such big programs in a meaningful way.

Another obstacle in debugging parallel programs is introduced by nondeterminism, which plays a bigger role in the parallel case than in sequential programs. This is due to the fact that in addition to the sources of nondeterminism that we know from sequential programs, there are several nondeterministic program constructs that are specific to parallel programs. Examples are wild-card receive function calls in message passing programs or unordered access to shared data in shared memory programs. The problems that arise from nondeterministic behaviour during the debugging process are severe, and are usually subsummed as probe effect, completeness problem, and irreproducibility effect [2]. For debugging the irreproducibility effect is the more problematic, since it inhibits cyclic debugging. For this reason several methods have been developed to enable deterministic re-executions, one of the first being the instant replay mechanism proposed by LeBlanc and Mellor-Crummey [3]. In principle all these replay approaches work in two phases. In the record phase, information about what happened during the program's execution is recorded and saved to stable memory. This information, the socalled trace of the program, is used in the second phase, the replay phase, where the program is re-executed under the control of a replay system. The recorded information is used to enforce the same decisions at nondeterministic events as in the original program run, hence permitting any number of equivalent re-executions of the program.

\subsection{Related Work}

Many existing debugging tools, commercial and non-commercail, for sequential and parallel programs, support some or all of the above described techniques. Well known examples for sequential debuggers are gdb and $\mathrm{dbx}$, all with similar and mature functionality. In the parallel case the situation is more wide-spread. Some approaches extend the sequential case and use multiple instances of a sequential debugger like gdb simultaneously [4 5]. This class of parallel debugger does not scale very well, and they do not address the pitfalls of nondeterminism. Therfore they are useful only for small deterministic programs.

Another class of parallel debuggers uses the record\&replay approach. Such debuggers can handle nondeterminism and hence can provide cyclic debugging. Some provide distributed checkpoints and breakpoints, which is useful for long running programs. Examples are [6, 7, 8]. Unfortunately they don't scale well on the process axes, i.e. they are difficult to use for massively parallel programs.

Our approach is in some sense similar to program slicing in general [9] and the work of Dusterwald et al. on slicing distributed programs in particular [10]. However, their work is based on control flow analysis and the distributed dependence graph, while we use the event graph (as described below) as fundament. Also, they don't support breakpoints and checkpoints, which is a big drawback for long running programs.

\section{Single Process Debugging}

In this section we describe our debugging strategy, which we call single-process debugging. The target applications of our work are massively-parallel message-passing 
programs. Without loss of generality we confine ourselves to SPMD message-passing programs written in the $\mathrm{C}$ programming language. For inter-process communication we use only two functions defined in the MPI-Standard [11], namely MPI_Send for sending, and MPI_Recv for receiving data.

There are several prerequisites which must be met for our approach to work. Firstly, we need to be able to replay parallel programs deterministically. We can do this in a very efficient manner using our Record\&Replay approach called RON. Furthermore we describe how we can isolate a process (or a group of processes) from a parallel program, in a sense, that we can (re-)execute this process stand-alone, without having to execute the entire parallel program. We use checkpoints as well as breakpoints; both techniques have to be adapted to work in the case of parallel programs. Finally we describe how we combine these techniques to the single process debugging strategy.

\subsection{Modelling Program Executions}

For analysis of the recorded trace data we need a formalism in order to be able to apply mathematical methods like graph operations on the data. As a formal representation of a program's execution we use the event graph model:

Definition Event Graph [12]. An event graph is a directed graph $G=(E, \rightarrow)$, where $E$ is the non-empty set of events $e \in E$, while $\rightarrow$ is a relation connecting events, such that $x \rightarrow y$ means that there is an edge from event $x$ to event $y$ in $G$ with the "tail" at event $x$ and the "head" at event $y$.

The events $e \in E$ are the recorded events observed during a program's execution, like for example send or receive events. The relation connecting the events of an event graph is the so called happened-before relation [13].

\subsection{Deterministic Replay}

A crucial aspect of any monitoring or Record\&Replay approach, respectively, is to keep the influence of the monitoring system on the program as low as possibe. Given the fact, that in MPI non-overtaking communication is guaranteed, one can show that it is sufficcient to record only the process-id of the corresponding sending process for every nondeterministic call to MPI_Recv. This can be done with very little effort during program execution and results in very small tracefiles. In subsequent replay iterations equivalent program runs are guaranteed by replacing every occurance of a wild-card receive with a deterministic receive, where the process-id of the message source is read from the previously recorded tracefiles. Once the execution path is fixed one can extend the amount of recorded data without running the risk to perturb the order of message reception. This Record\&Replay mechanism has been implemented in the monitoring tool NOPE [14], which is part of the DeWiz debugging environment [15].

With deterministic replay we are now able to do cyclic debugging. However, as stated before, due to the size of massively-parallel programs, cyclic debugging is by far not sufficient as debugging strategy. The next step is therefore to reduce the amount of analysis data. We can do this by narrowing the investigated parts of the program in two dimensions, namely along the process axes using process isolation, as well as along the time axes using breakpoints and checkpoints. 


\subsection{Process Isolation}

During debugging of a massively-parallel program the user might get the suspicion that there is a bug on a certain process. At this point, one would start the program again, possibly several times, paying special attention on that particular process. During all these debugging iterations the whole program, which consists of many processes, must be executed all the times, although the user focuses only on one or a few processes. This has many disadvantages, it wastes CPU time, which is a cost issue, unneccessary trace data is generated (and possibly analysed in vain), and so on. Therefore we use the idea of process isolation, where we "isolate" one process (or a group of processes) from the rest of the parallel program, making them executable stand-alone.

Isolating process $P_{i}$ from its context requires three steps:

1. Initial execution of the entire program: generation of trace data.

2. Build the event graph.

3. Isolation step: re-execute process $P_{i}$ and all (directly or indirectly) dependent processes. Save the contents of all incoming messages on process $P_{i}$.

During subsequent isolated executions of $P_{i}$ incoming messages are not received by MPI_Recv, but the contents of the message is read from the previously generated tracefiles. The number of processes that have to be re-executed in step 3 is not fixed, but depends on the program being analysed. Based on the event graph we use a recursive algorithm to determine which processes have to be re-executed.

\subsection{Breakpoints in Parallel Programs}

Distributed breakpoints (and checkpoints) are used to reduce the program's complexity along the time axis. A distributed breakpoint is a set of sequential breakpoints, one on each process of the parallel program: $B_{i}=\left(S_{i, 0}, \ldots, S_{i,(n-1)}\right)$, with $n$ being the number of processes in the parallel program. In our approach we give the user two possibilities to define a distributed breakpoint: manually, by modifying the source code, or semiautomatically, based on the event graph.

Using the manual approach the user can instert special breakpoint code at any position in the source code. However, in this case it lies in the responsibility of the user that this code is placed properly so that the sequential breakpoints are reached by every process during program execution (which of course is crucial for a distributed breakpoint to be useful).

The second possibility to specify a distributed breakpoint is situated on a higher level of abstraction (or program representation) compared to the source code level. A recorded program run, or rather the event graph, can be visualized as space-time diagram, where - amongst others - there are send and receive events as nodes in the graph, which are pairwise connected by arcs. The user can select any event $e_{i, p}$ (the $i^{\text {th }}$ event on process $p$ ) in the graph by simply clicking on it; this event will be the origin of the distributed breakpoint. After selection of such an event, we can automatically determine which events on all other processes have to be part of the distributed breakpoint, by using the happened-before relation. 


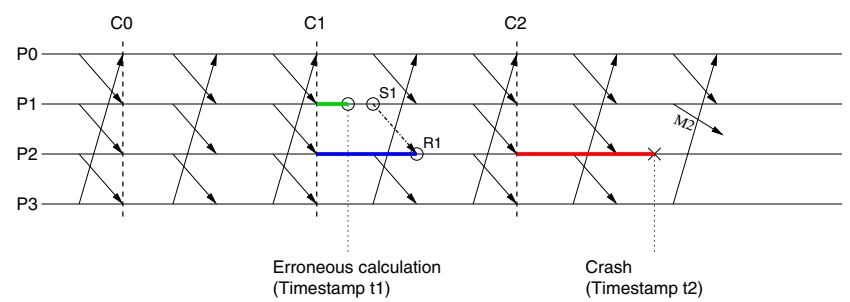

Fig. 1. Example debugging scenario

\subsection{Checkpoints in Parallel Programs}

Another approach to reduce the cycle time of the debugging iterations is to use checkpointing. Similar to a distributed breakpoint a distributed checkpoint consists of one sequential checkpoint for each process of the parallel program, which form a so called checkpoint line. An important aspect is the placement of checkpoints, which can be done either semi-automatically or automatically. The semi-automatic approach is done via the visual representation of the event graph of the parallel program. To place a checkpoint the user can select any arbitrary point on one process, the corresponding checkpoints on the remaining processes are calculated automatically, based on the event graph. During the next replay of the program at all user specified checkpoint locations the current state of the process is saved to stable storage.

The semi-automatic approach has several disadvantages, namely placing checkpoints on a regular base would become a tedious task. Therefore we have the possibility to place checkpoints automatically. In principle each process saves it's state periodically to stable storage. However, several requirements must be met to ensure so-called consistent checkpoints, e.g. one must take care of messages that would cross a checkpoint line, and others. Details on that can be found in [16].

\subsection{Putting It All Together}

All these techniques have been applied in debugging programs, sequential and parallel as well. Due to the difficulties introduced by massively-parallel programs as described in section 2.2 these techniques are limited to relatively small parallel programs. Therefore we have tried to combine them in a proper way, giving the user the possibility to handle even the biggest parallel programs.

The two main ideas are to keep the user's focus only on relevant parts of the program on the one hand, i.e. on parts where errors are suspected, and on the other hand to save computing resources during debugging, which is often an important cost factor. We can achieve this by re-executing only small parts of the massively parallel program during the debugging cycles. The following simple scenario in Figure11illustrates the idea.

1. A small parallel program consisting of four processes $P_{0}$ to $P_{3}$ was executed and crashed after a while, the user starts a debugging session. Unless the initial execution was not monitored, the user would initiate a monitored re-execution in order to permit deterministic re-executions and to generate trace data. Also checkpoints are generated periodically. 
2. The event graph of the previous execution is generated and visualized as space-time diagram, as displayed in Figure 1 We see message exchanges indicated by arrows, and three distributed checkpoints $C_{0}$ to $C_{2}$.

3. The user suspects a crash on $P_{2}$ at timestamp $t_{2}$ (based on various indications, eg. error messages from the MPI environment, or - in our example - by the fact that message $M_{2}$ is pending, etc.), therefore debugging starts on $P_{2}$. The cause of the crash is an erroneous calculation on process $P_{1}$ at timestamp $t_{1}$, which of course is unknown to the user at that time.

4. In a first step only a small part of $P_{2}$ is selected and debugged: $P_{2}$ is isolated starting at the closest checkpoint to $t_{2}$, in our case $C_{2}$. The small section $\left[C_{2}: t_{2}\right]$ can now be re-executed and inspected any arbitrary number of times stand alone.

5. Since the cause of the crash could not be found in section $\left[C_{2}: t_{2}\right]$, the investigated interval is extended to $\left[C_{1}: t_{2}\right]$, again using process isolation, etc. Here the user finds that an erroneous value is received at receive event $R_{1}$. Therefore the debugging session is continued on process $P_{1}$ in the interval $\left[C_{1}: C_{2}\right]$, since this is the location of the corresponding send event $S_{1}$. For that purpose process $P_{1}$ must be isolated and re-executed in the mentioned interval.

6. Finally the user finds the location of the erroneous calculation on process $P_{1}$ at timestamp $t_{1}$, which caused the crash later on $P_{2}$.

It is important to note that all steps necessary for process isolation, all transitions from one process to another, etc. can be performed automatically and transparently in the background. The user simply selects a (small) region on a process for debugging, and this section is isolated automatically. In case of the reception of an erroneous value as in the example above, the user just initiates a switch to the sending process; which section (or checkpointing interval, respectively) on the sending process has to be isolated can be calculated automatically using the relations in the event graph.

\section{Conclusion and Future Work}

We have presented a scalable debugging strategy for massively parallel programs. The main idea is to narrow the range of the investigated parts of the program during debugging in an intelligent way, both in time and in space. This keeps the focus of the user on the relevant parts of the program, and, since re-execution during debugging is much less ressource intensive, may actually save money.

We are working on several improvements of this approach. For example, the integration of control flow analysis could improve the understanding of error propagation in the program, hence making the tracking of errors more efficient. Several work has been done in this field, for example the work on slicing parallel programs described in [10]. Also an extension to shared memory programs and distributed programs in general seems useful and will be investigated further.

\section{References}

1. Foster, I., Kesselman, C., Tuecke, S.: The anatomy of the grid: Enabling scalable virtual organizations. The International Journal of High Performance Computing Applications 15 (2001) 200-222 
2. Schaubschläger, C.: Automatic testing of nondeterministic programs in message passing systems. Masters Thesis, GUP, Johannes Kepler University, Linz, Austria. http://www.gup.unilinz.ac.at $/{ }^{\mathrm{cs}} /$ thesis (2000)

3. LeBlanc, T.J., Mellor-Crummey, J.M.: Debugging parallel programs with instant replay. IEEE Trans. Comput. 36 (1987) 471-482

4. Balle, S.M., Brett, B.R., Chen, C.P., LaFrance-Linden, D.: Extending a traditional debugger to debug massively parallel programs. Journal of Parallel and Distributed Computing 64 (2004) 617-628

5. Cunha, J., Lourenco, J., Antao, T.: A debugging engine for parallel and distributed environment (1996)

6. Kacsuk, P.: Systematic macrostep debugging of message passing parallel programs. Future Gener. Comput. Syst. 16 (2000) 609-624

7. Etnus: Totalview debugger. http://www.etnus.com/ (2005)

8. Absoft, Corp.: DDT - Distributed Debugging Tool (2005)

9. Weiser, M.: Program slicing. In: ICSE '81: Proceedings of the 5th international conference on Software engineering, Piscataway, NJ, USA, IEEE Press (1981) 439-449

10. Duesterwald, E., Gupta, R., Soffa, M.L.: Distributed Slicing and Partial Re-execution for Distributed Programs. In: Languages and Compilers for Parallel Computing. (1992) 497-511

11. Message Passing Interface Forum: MPI: A Message-Passing Interface Standard - Verion 1.1. http://www.mcs.anl.gov/mpi/ (1995)

12. Kranzlmüller, D.: Event graph analysis for debugging massively parallel programs. $\mathrm{PhD}$ thesis, GUP, Joh. Kepler Univ. Linz. http://www.gup.uni-linz.ac.at/ /dk/thesis (2000)

13. Lamport, L.: Time, clocks, and the ordering of events in a distributed system. In: Communications of the ACM, Vol. 21, No. 7. (1978) 558-565

14. Kranzlmüller, D., Volkert, J.: Nope: A nondeterministic program evaluator. In: Proc. of ACPC99, 4th Intl. ACPC Conference, LNCS, Vol. 1557. (1999) 490-499

15. Kobler, R., Schaubschläger, C., Aichinger, B., Kranzlmller, D., Volkert, J.: Examples of monitoring and program analysis activities with dewiz. In: Proc. DAPSYS 2004 (5th AustrianHungarian Workshop On Distributed And Parallel Systems). (2004)

16. Thoai, N.: Checkpointing techniques for minimizing the waiting time during debugging long-running parallel programs. PhD. Thesis, GUP, Johannes Kepler University, Linz, Austria (2003) 